\section{Silent Hypoxia in COVID-19: What is Old is New Again!}

Sir,

With Coronavirus disease of 2019 (COVID-19) pandemic, silent hypoxemia (significant hypoxemia without dyspnea) is a phenomenon to understand with associated shunt physiology, preserved lung compliance, and lack of dead space. ${ }^{1}$ It applies to any lung pathology which causes a reserved amount of shunt while saving the rest of the lung such as lobar pneumonia or atelectasis. Silent hypoxemia can last forever but individuals could present to a healthcare facility with this condition occasionally. COVID-19 made us to think about this forgotten approach.

COVID-19 lung injury includes viral medial inflammation, alveolar epithelial dysfunction, impaired surfactant, alveolar collapse, and increased pulmonary artery pressure patients. ${ }^{1,2}$ The disease process is been treated as acute respiratory distress syndrome (ARDS) but it is not typical ARDS. With normal lung compliance and many of them not in respiratory distress, hypoxemia does not correlate with their clinical condition. ${ }^{1-2}$ Patients often presents in stable condition where they are able to speak full sentences with oxygen saturation of $80 \%$, hence pulse oximetry may be a marker for respiratory distress. $^{1,2}$

Intra-pulmonary shunting, relatively preserved lung compliance, lack of excessive dead space, lack of substantial pulmonary embolism or microvascular thrombosis and dysfunctional hypoxemic vasoconstriction are the key features. ${ }^{1,2}$ COVID-19 patients faced slow onset severe hypoxemia with hypocapnia. Atelectasis, obesity, elderly and underlying structural lung disease could be the predisposing factors for rapid deterioration in the clinical condition. ${ }^{1,2}$ COVID-19 related hypoxemia is different as patient is not subjectively tachypneic but patterns of respiration are making patients to have an enhanced respiratory drive and minute ventilation. ${ }^{1,2}$ In compensation, patients have increased carbon dioxide $\left(\mathrm{CO}_{2}\right)$ clearance which keeps them away from getting short of breath. ${ }^{1,2}$

Stages of silent hypoxia described as zones divided into subclinical, detection and high mortality. ${ }^{1,2}$ Subclinical zone is where oxygen saturation is normal with normal respiratory rate. Detection zone is where there is gradual decline in oxygen saturation with no subjective shortness of breath but respiratory rate started to rise with progressive self-inflicted lung injury (SILI). This is the zone for intervention; and dramatic results can be seen if treatment is initiated. ${ }^{1,2} \mathrm{High}$ mortality is the zone where oxygen saturation drops with increasing subjective shortness of breath. This is the zone where type $\mathrm{H}$ physiology transition with respiratory failure can occur. ${ }^{1}$

Silent hypoxemia can become a severe form of hypoxemia when there is a poor response to supplemental oxygen. This had been seen in aviation science ${ }^{3,4}$; and COVID-19 made us to rethink about this physiology. Firstly, with oxygenation, hypoxemia occurs when blood from right ventricle goes into left ventricle without being oxygenated. ${ }^{1,2,5}$ It is due to ventilation perfusion mismatch; hence, the net result is hypoxemia. Increasing inhaled oxygen concentration can be used here. Secondly there is the shunt physiology such anatomicabnormalities or dysfunctional parts of lung ${ }^{1-4}$ Any patient with desaturation despite high supplemental oxygen likely has a shunt. $\mathrm{CO}_{2}$ clearance rely on amount of gas exchange with key reliance on dead space such as scarred alveoli due to ARDS or in case pulmonary embolism where there is gas exchange but no $\mathrm{CO}_{2}$ clearance..$^{1-3}$ Increasing dead space means patient must inhale more gas to maintain the same $\mathrm{CO}_{2}$. Silent hypoxemia with normal work of breathing occurs when there is right to left shunt, no excessive dead space, normal lung compliance and lung resistance. ${ }^{3,4}$

Management of silent hypoxemia in COVID-19 does not require intubation. Personal protection equipment should be the initial consideration. Treatment options included high flow nasal cannula (HFNC) with surgical facemask over patient's mouth, awake-proning and repositioning (left lateral, sitting and right lateral) to improve the saturation to $90 \% .{ }^{3,4}$ Awake proning showed feasibility in the times of scarce resources and shortage of ventilators. Nasal cannula with oxygen saturation goal of $88-92 \%$ is been reported in the literature. Continuous positive airway pressure therapy (CPAP) is another option before intubation. Airway pressure release ventilation (APRV), conventional low tidal-volume ventilation, permissive hypercapnia and optimization of metabolic acid/base status and inhaled pulmonary vasodilators are further treatment modalities. ${ }^{4,5}$

\section{CONFLICT OF INTEREST:}

Authors declared no conflict of interest.

\section{AUTHORS' CONTRIBUTION:}

SS, MAB: Substantial contributions to the design of the work; drafting the work and revising it critically for important intellectual content; approved the final version to be published.

\section{REFERENCES}

1. Salim Rezaie. "COVID-19 Hypoxemia: A Better and Still Safe Way", REBEL EM blog, March 31, 2020. Available at: http://rebelem.com/covid-19-hypoxemia-a-better-andstill-safe-way/

2. Scott Weingart. EMCrit 189 - Secret Sixth Cause of Arterial Hypoxemia and Vasoactive Purity. EMCrit Blog. Published on December 28, 2016. Accessed on May 4th 2020. Available at [http://emcrit.org/emcrit/ end-yeargrab-bag/].

3. West J. American Medical Research Expedition to Everest. High Alt Med Biol 2010; 11(2):103-10.

4. Izraeli S, Avgar D, Glikson M, Shochat I, Glovinsky Y, Ribak J. Determination of the "time of useful consciousness" (TUC) in repeated exposures to simulated altitude 
of 25,000 ft (7,620 m). Aviat Space Environ Med 1988; 59(11 Pt 1):1103-5.

5. Gattinoni L, Chiumello D, Caironi P, Mattia Busana M, Romitti F, Brazzi L, et al. COVID-19 pneumonia: Different respiratory treatments for different phenotypes? Intensive Care Med 2020; 46(6):1099-1102.

Sadaf Sheikh ${ }^{1}$ and Muhammad Akbar Baig ${ }^{2}$

${ }^{1}$ Department of Emergency Medicine, Sultan Qaboos University Hospital, Muscat, Oman
${ }^{2}$ Department of Emergency Medicine, The Aga Khan University Hospital, Karachi, Pakistan

Correspondence to: Dr. Sadaf Sheikh, Department of Emergency Medicine, Sultan Qaboos University Hospital, Muscat, Oman

E-mail: sheikh.sadaf@gmail.com

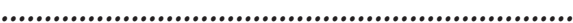

Received: May 15, 2020; Revised: June 10, 2020;

Accepted: June 14, 2020

DOI: https://doi.org/10.29271/jcpsp.2020.JCPSPCR.CR70 